\title{
A Grey Box Neural Network Model of Basal Ganglia for Gait Signal of Patients with Huntington Disease
}

Abbas Pourhedayat ${ }^{1}$, Yashar Sarbaz ${ }^{1 *}$

1. Department of Mechatronics Engineering, School of Engineering-Emerging Technologies, University of Tabriz, Tabriz, Iran.

Citation: Pourhedayat, A., \& Sarbaz, Y. (2016). A grey box neural network model of basal ganglia for gait signal of patients with huntington disease. Basic and Clinical Neuroscience, 7(2), 107-114. http://dx.doi.org/10.15412/J.BCN.03070204

http://dx.doi.org/10.15412/J.BCN.03070204

Article info:

Received: 06 April 2015

First Revision: 29 May 2015

Accepted: 23 July 2015
Key Words:

Basal ganglia,

Huntington disease,

Neural network models,

Neurotransmitters

\begin{abstract}
A B S T RA C T
Introduction: Huntington disease (HD) is a progressive neurodegenerative disease which affects movement control system of the brain. HD symptoms lead to patient's gait change and influence stride time intervals. In this study, we present a grey box mathematical model to simulate HD disorders. This model contains main physiological findings about BG.

Methods: We used artificial neural networks (ANN) and predetermined data to model healthy state behavior, and then we trained patients with $\mathrm{HD}$ with this model. All blocks and relations between them were designed based on physiological findings.

Results: According to the physiological findings, increasing or decreasing model connection weights are indicative of change in secretion of respective neurotransmitters. Our results show the simulating ability of the model in normal condition and different disease stages.

Conclusion: Fine similarity between the presented model and BG physiological structure with its high ability in simulating HD disorders, introduces this model as a powerful tool to analyze HD behavior.
\end{abstract}

\section{Introduction}

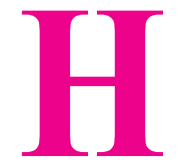

untington disease (HD) is a progressive neurodegenerative disease which affects movement control system of the brain. Degeneration of the Basal Ganglia (BG) of the brain causes movement disorders as it regulates movements and makes them smooth. Movement symptoms significantly appear in limbs and include chorea, ballism, and athetosis.

Ballism refers to rapid flailing movements. Chorea includes very similar excessive, involuntary movements, like those of ballism, but less abrupt and wild. Athetosis is slow writhing movements of fingers, hands, and sometimes toes. HD symptoms lead to patient's gait change and influence stride time intervals, the gait speed, and rhythmicity of walking (Kandel, Schwartz, \& Jessell, 2000). In this respect, researchers use these changes to study HD.

In 1998, Hausdorff et al. studied the gait variability and BG disorders. They focused on the stride times of patients with HD and Parkinson disease (PD). They showed that gait variability significantly increases in these patients and concluded that the variability degree correlates with disease severity (Hausdorff et al., 1998). They already affirmed that

* Corresponding Author:

Yashar Sarbaz, PhD

Address: School of Engineering-Emerging Technologies, University of Tabriz, Tabriz, Iran.

Tel: +98 (411) $3393754 \quad$ Fax +98 (411) 3294626

E-mail: yashar.sarbaz@tabrizu.ac.ir 
in HD, patients' stride time intervals are not correlated and stable (Hausdorff et al., 1997). For several years, no mathematical analysis was implemented on HD. In 2006, Aziz and Arif started to study gait complexity of patients with neurodegenerative disease. They used threshold dependent symbolic entropy, based on analyzing symbolic nonlinear time series to study this complexity. Their research showed a significant distance for a certain range of thresholds between control and neural disease subjects. They had also investigated the complexity of physiological signal and randomized noisy data. They found that at low threshold values, the physiological signal's complexity is higher than random signals (Aziz \& Arif, 2006).

To study behavior of patients with HD, Sarbaz et al. introduced a mathematical model of BG. They presented this model based on BG structure to simulate abrupt movements in patients with HD. In each block, they used neuron membrane relations (Sarbaz, Banaei, \& Gharib-Zadeh, 2007). One year later, Liao et al. scrutinized asymmetry of gait signals in patients with HD versus normal persons by multiresolution entropy analysis. Finally, they found that gait symmetry in patients with PD, HD, and Amyotrophic Lateral Sclerosis (ALS) is significantly disturbed (Liao, Wang, $\& \mathrm{He}, 2008)$. In the same year, Banaie et al. introduced another mathematical model to study gait behavior in patients with HD. The presented model concluded more physiological information in comparison with Sarbaz model (Sarbaz et al., 2007).

Moreover the model simulated neurotransmitters role in the BG and could evaluate possible drug treatments (Banaie, Sarbaz, Gharib-Zadeh, \& Towhidkhah, 2008). In 2009, Dutta et al. managed to separate patients with neurological disorders like HD, PD, and ALS. They achieved 87.1\% accuracy using by Elman's recurrent neural network and extracted features from stance, swing, and double support intervals (Dutta, Chatterjee, \& Munshi, 2009). Likewise, in another research, Zheng et al. used machine learning and statistical approaches to distinguish patients with HD, PD, and ALS based on gait analyses. For this purpose, they utilized 3 supervised classification methods (Support Vector Machine, K-Star, and Random Forest) with some extracted features from gait cycles. The results demonstrated the feasibility of applied computational classification techniques in characterizing these 3 diseases (Zheng, Yang, Wang, \& McClean, 2009). In 2011, Merrikh-Bayat exerted time series analysis on some neurodegenerative disorders like HD. Results showed that the average dimension of patients with PD, HD and ALS are more unfavorable than healthy control subjects (Merrikh-Bayat, 2011). Furthermore, Banaie et al. proceeded to extract proper features for discriminating patients with $\mathrm{HD}, \mathrm{PD}$, and ALS based on their gait signals.
The outcome showed that quadratic, bayes classifier had better result in classifying patients (Banaie, Pooyan, \& Mikaeili, 2011) and in another study, Sarbaz et al. proposed a new model for healthy and patients with PD; They also introduced a new index for PD severity (Sarbaz, Gharib-Zadeh, Towhidkhah, Banaie, \& Jafari, 2011). They presented a model for basal ganglia structure to generate stride time interval signal in model output for healthy and PD states; Their model had high ability in simulating normal and patient cases (Sarbaz, Towhidkhah, Banaei, Pooyan, \& Gharib-Zadeh, 2011). Next year, Daliri showed that features derived from double support intervals are common effective features for the diagnosis of neurodegenerative diseases using the gait dynamics (Daliri, 2012). Finally in 2013, Sarbaz and Pourhedayat separated patients with HD from normal persons using Artificial Neural Network (ANN) classifier using extracted features from power spectra density. Their best separation accuracy was $96.6 \%$ (Sarbaz \& Pourhedayat, 2013); Several studies like this had already published about PD separation (Sarbaz, Towhidkhah, Gharib-Zadeh, \& Jafari, 2012; Sarbaz, Towhidkhah, Mosavari, Janani, \& Soltan-Zadeh, 2013).

Various studies tried to quantify HD movement disorders. Meanwhile some others presented mathematical models of HD behavior. In this study, we present a grey box mathematical model to simulate HD disorders. This model contains main physiological findings about BG. Presenting such a model could help us to understand the brain performance, HD behavior, and the reason of movement disorders in patients with HD.

\section{Materials \& Methods}

\subsection{Physiological background}

The basal ganglia plays a major role in movement control (Galvan \& Smith, 2010) and receives input from cortex and sends output via thalamus to supplementary motor areas (Kliem \& Wichmann, 2009). BG includes 5 neural blocks. Internal parts are putamen and caudate nucleus (named striatum) and other parts are known as globus pallidus (GP) and substantia nigra (SN). GP is divided into internal (GPi) and external (GPe) segments. Similarly, SN contains compacta $(\mathrm{SNc})$ and reticulate $(\mathrm{SNr})$ parts. GPi and SNr blocks terminate the movement control segment. The BG included two main pathways between blocks which starts from striatum and leads to the BG output block. Primary information processing is done in direct pathway. Indirect pathway connects input and output of the BG via GPe and subthalamic nucleus (STN). This path possesses more important role in movement control because it processes more information 
compare to the direct one (Fenneya, Jogb, \& Duval, 2008). $\mathrm{BG}$ parts and their connections are shown in figure 1.

The balance of two pathways is tuned by SNc modulatory effects. On the other hand, dopamine and internal parts of striatum control the BG behavior. Some cholinergic and GABA-ergic neurons of striatum are destroyed in HD. This damage causes overactivation in the direct pathway and underactivation in the other one. The neurons death in striatum decreases GPe and $\mathrm{SNr}$ inhibition. This in turn results in higher GPe activation which inhibits STN much more than a lesion and has a similar effect on silencing STN. Finally, these damages lead to decrease in the output of BG to thalamus so as the BG tuning role on movements, decreases and HD disorders appear in patients (Vonsattel, Keller, \& Amaya, 2008). Changes in connection weights are shown in Figure 2. BG disorders deteriorate with HD progress and affect patient's gait and movement.

\subsection{Clinical data}

In this study, we used predetermined data taken from http://www.physionet.org. The dataset contains gait signal of 20 patients with HD and 16 healthy persons. Each sample has 5 minutes recorded signal of stride, swing, and stand times for each leg and double support signal for both legs. An expert physician labelled patients' states from 0 to 13 (0 equal to the most severe state and 13 for a healthy one). To measure time intervals, force sensors had been embedded in participant's shoes which recorded 300 samples per second. First 20 seconds of records were deleted to reduce initial oversight (Hausdorff et al., 1998).

\subsection{Mathematical model structure}

Neurons death in some BG parts causes abnormal changes in the amount of neurotransmitters between connections. Physiological studies suggest that these abnormal changes are the main cause of HD. Our mathematical model is designed based on BG structure and its principle effect on HD. All of input and output connections among BG parts are modeled similar to its structure (Figure 3). Each BG block contains numerous parallel neurons and we tried to present a matched mathematical relation for each one of them. ANN is known as a proper mathematical tool in modeling neuron and central nervous system's behavior (Mandic \& Chambers, 2001) and can properly simulate brain neural blocks. Each block shows a part of BG with the same name.

Similar to BG structure, we have two paths in the model; Direct pathway which connects striatum to GPi/SNr and indirect pathway that starts from striatum, crosses GPe and subthalamic nucleus, and finishes in GPi/SNr. So we have two feedbacks in the model; one in subthalamic nucleus to GPe input and the other in SNc to striatum. All blocks have 10 neurons except output; This number is set after examinations to achieve acceptable accuracy with fewer computations.

From physiological perspective, we know that cortex input for gait is without any pattern and duration so these factors influence on person's gait via BG. To simulate this process in the model and to be ensured that BG makes pattern, random signal is used in input block.

For each BG block, we used the single layer feed-forward neural network that models various parallel neurons in the block (Too, 1999). Model structure of each block is exhibited in Figure 4 and its relations are as follows:

Exposed model was trained for healthy state to find proper weights using back-propagation method. The found weights simulated neurotransmitters in BG connections. Moreover, a neurologist should definitely determine the inhibitory or excitatory behavior of a connection. We considered these

$$
\text { output }=f\left\{\left(\sum_{i} w_{i}^{*} I\right)+b\right\} \quad f(x)=\frac{e^{x}-e^{-x}}{e^{x}+e^{-x}}
$$

types in our model to reach a realistic one. Therefore, weights were assumed positive in excitatory connections and negative in inhibitory ones. From physiological viewpoint, the presented model can simulate the real BG behavior in HD disorders. Therefore, to find connection weights in disease states, normal weights were adapted similar to changes which are caused in HD state (Figure 2).

\section{Results}

Figure 5 shows a real signal and the model response in healthy state. In the database, a parameter shows the severity of disease. Severity parameter decreases when disease progress intensifies brain destruction. Thus, we adapted model weights in different disease states. Our selected severities to show disease progress were $11,8,5$, and 2 whereas reported severities in the database ranged from 13 to 0 Model responses in 4 disease states with clinical recorded signals are shown in Figure 6.

According to the physiological findings, increasing or decreasing model connection weights are indicative of change in secretion of respective neurotransmitters. In each layer, we have some neurons that their output connection weights behave similar to the output of neurotransmitter amount To evaluate the amount of neurotransmitter in the output of each block, we need to define a proper matrix norm Although in linear algebra several different norms are presented, we found the Euclidean norm better than others in 


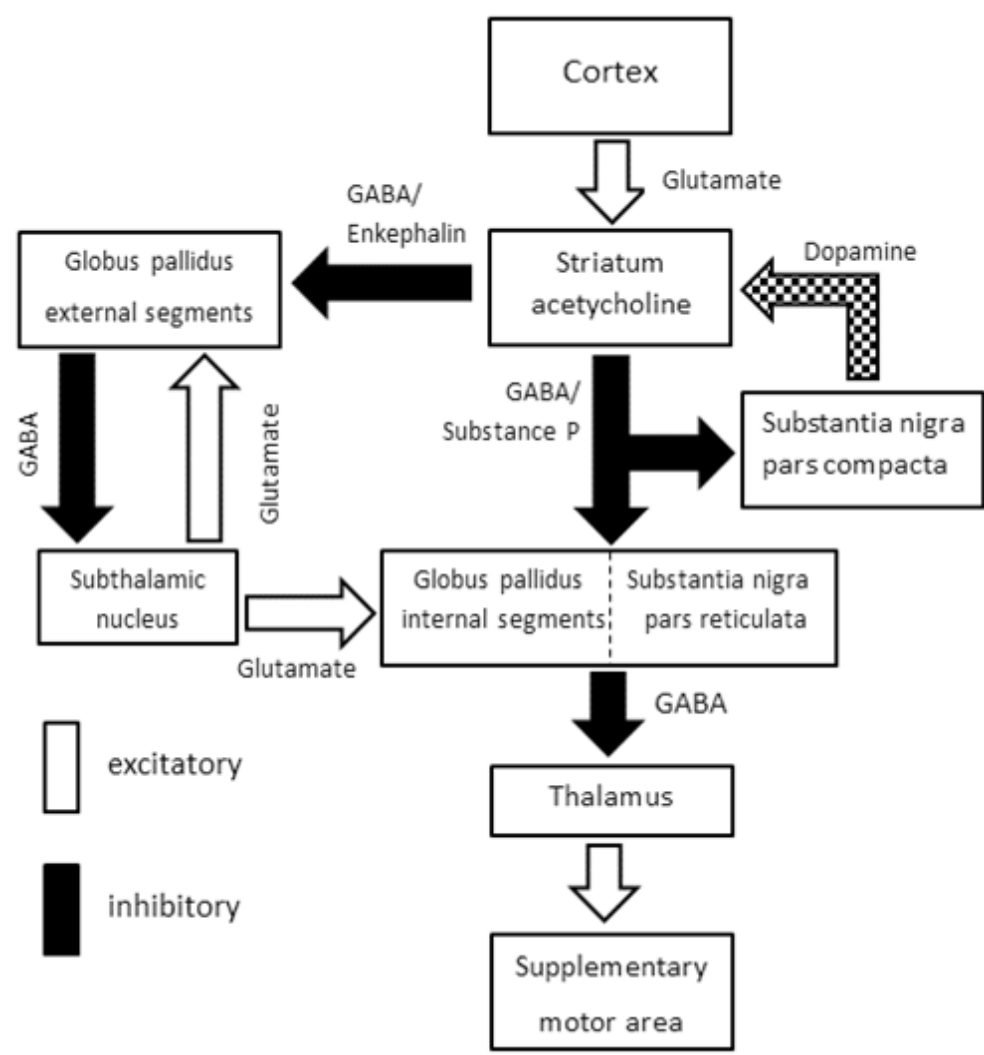

Figure 1. BG parts and connections among its blocks.

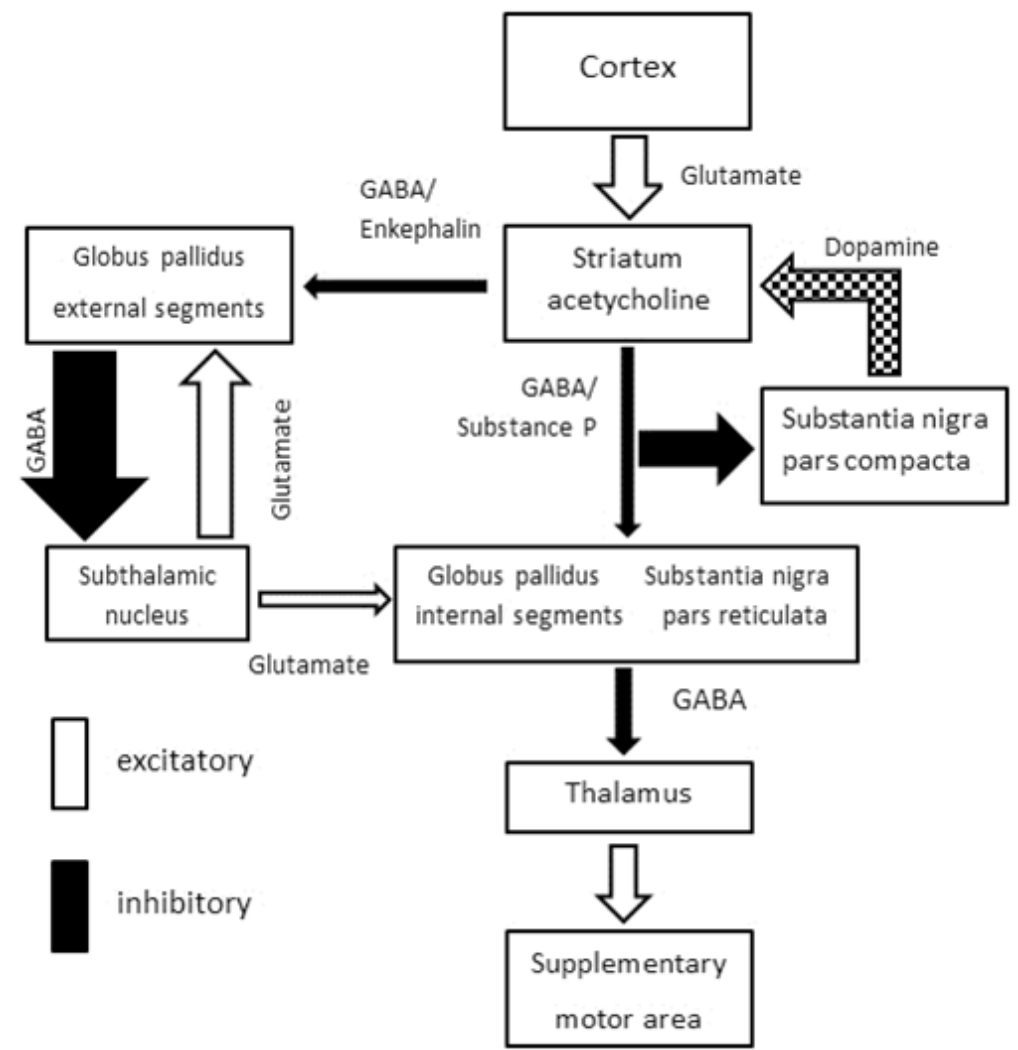

Figure 2. Connection weights changes of the BG in HD stages. 


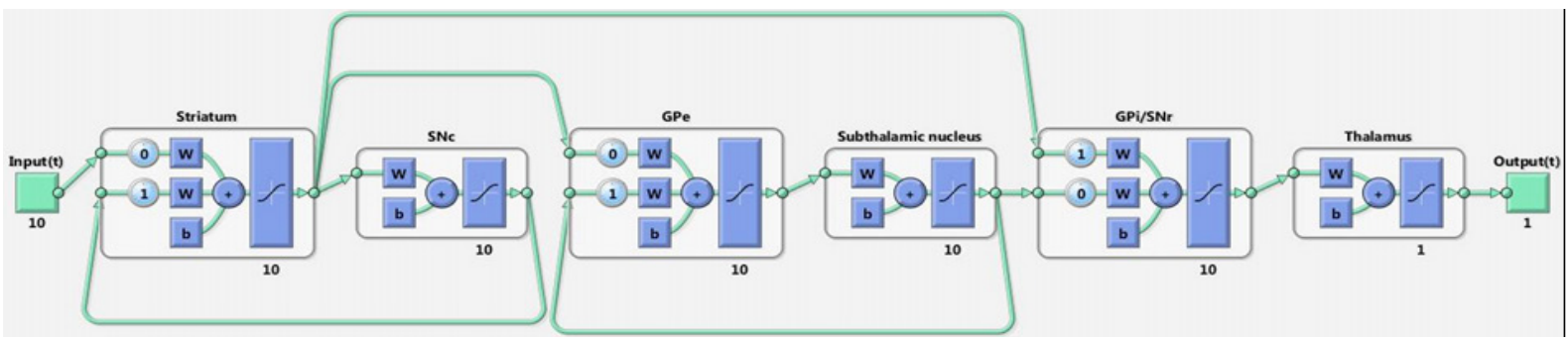

Figure 3. The structure of the presented model.

NEUR:SCIENCE

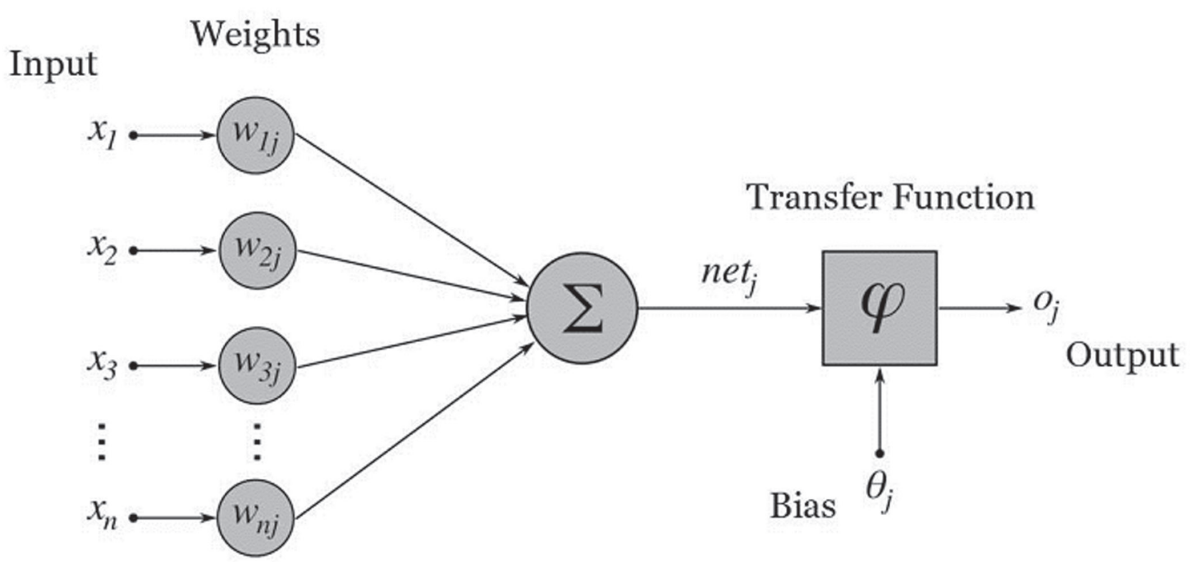

Figure 4. The structure of each block in our model.
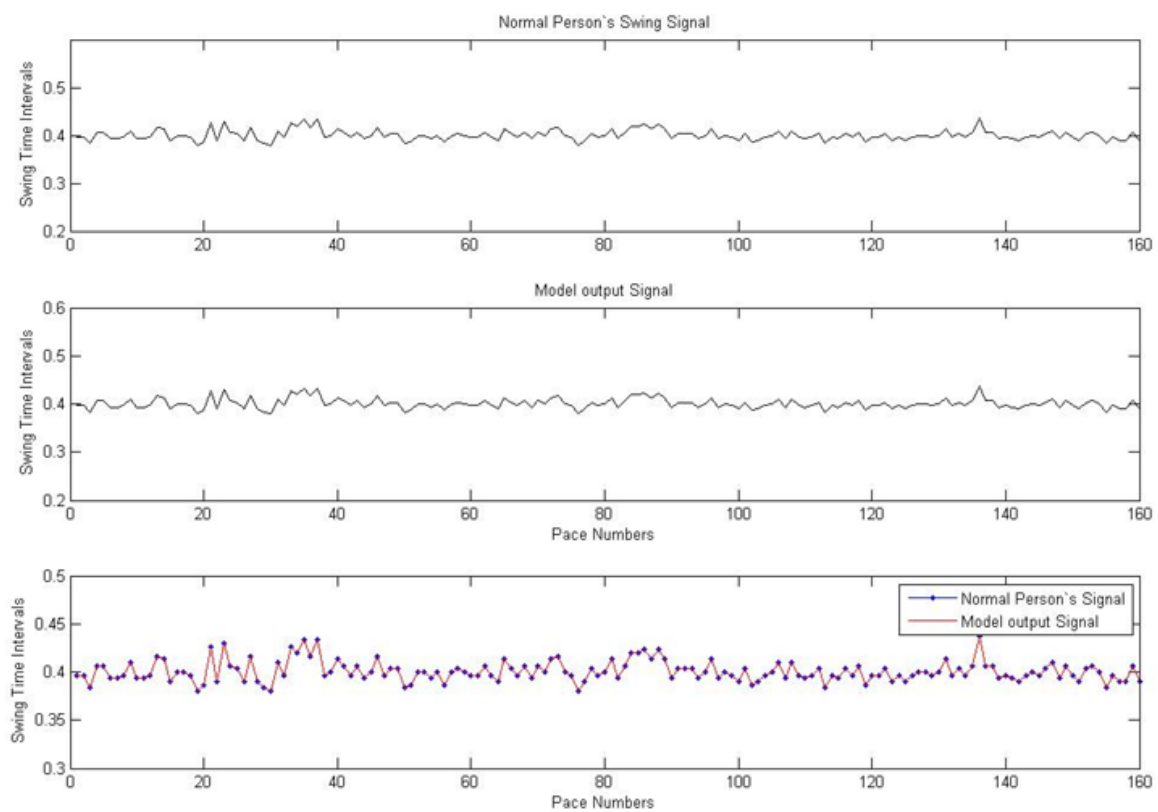

Figure 5. A real signal and the model response in healthy state. 

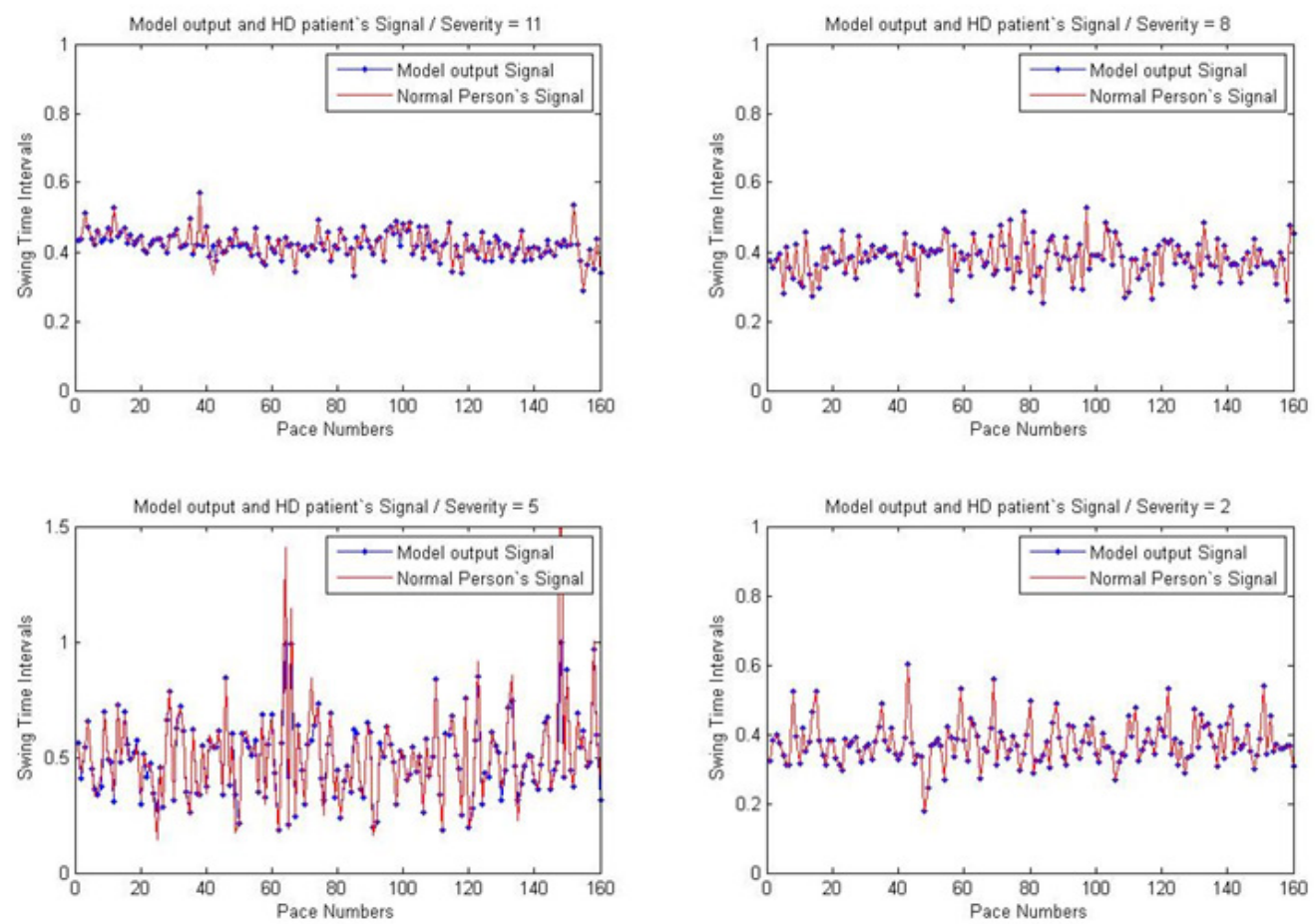

Figure 6. Model responses in 4 stages of disease with clinical recorded signals.

NEUR:SCIENCE

matching with the physiological behavior. This norm is defined as:

$$
\|M\|_{E}=\left[\sum_{i} \sum_{j} a_{i j}^{2}\right]^{1 / 2}
$$

Where $\mathrm{a}_{\mathrm{ij}}$ refers to the connection weight from $\mathrm{i}^{\text {th }}$ to $\mathrm{j}^{\text {th }}$ neurons. It is obvious that the summation of connection weights in each layer indicates the amount of neurotransmitter in related one. Table 1 shows neurotransmitters changes in BG parts in 4 disease states.

\section{Discussion}

$\mathrm{HD}$ is a progressive neurodegenerative disease with disabling conditions in final stages. Understanding the exact brain performance is impossible due to its complex structure which this complexity increases in disease state caused by brain destruction. Therefore, it is not clear why destruction of some specific BG neurons causes vast movement disorders in HD. In this regard, presenting mathematical models of brain's physiological performance plays a major role in increasing medical knowledge and shedding light on the performance of brain parts. Scrutiny mathematical models can be helpful in studying HD and expand our knowledge about HD states.

Although, neurological diseases like HD have been investigated by some researchers in various studies, few mathematical models have been presented for HD. In this study, we tried to present a complete mathematical model based

Table 1. Neurotransmitter changes of BG parts in four disease stages versus normal state.

\begin{tabular}{|c|c|c|c|c|c|c|c|}
\hline Weight changes & $\begin{array}{c}\text { SNc to } \\
\text { striatum }\end{array}$ & $\begin{array}{c}\text { Striatum to } \\
\text { GPi/SNr }\end{array}$ & $\begin{array}{c}\text { Striatum to } \\
\text { GPe }\end{array}$ & GPe to STN & STN to GPe & $\begin{array}{c}\text { STN to GPi/ } \\
\text { SNr }\end{array}$ & $\begin{array}{c}\text { GPi/SNr to } \\
\text { thalamus }\end{array}$ \\
\hline Disease stage 1 (severity: 11) & 0.160 & -0.077 & -4.618 & 7.262 & -3.441 & -0.640 & -2.566 \\
\hline Disease stage 2 (severity: 08) & 0.173 & -0.555 & -6.699 & 8.081 & -3.441 & -4.219 & -2.803 \\
\hline Disease stage 3 (severity: 05) & 0.173 & -0.903 & -9.175 & 13.351 & -3.441 & -5.586 & -3.351 \\
\hline Disease stage 4 (severity: 02) & 0.352 & -1.072 & -9.919 & 17.831 & -3.441 & -7.121 & -4.035 \\
\hline
\end{tabular}


on the main physiological findings which can simulate patient's gait (stride time intervals) with high accuracy. The model is designed based on the BG physiological structure. All BG parts and their input and output signals were arranged like biological neural blocks. Model blocks have excitatory or inhibitory connections according to real BG connections. To make internal blocks, a structure was envisaged with numerous parallel neurons like biological ones which could implement by ANN. Back-propagation method was applied to find proper weights for healthy state. Obtained weights were adapted with disease states to introduce a model of BG while the disease progresses. Our results show the simulating ability of the model in normal condition and different disease stages (Figures 5 and 6).

Based on physiological background, direct and indirect pathways are changed in HD states. This change increases in pathways as disease progresses due to destruction of BG. Related connection weights decrease against destruction accretion. In disease state due to striatum demolition, STN enhances output whereas GPe input from indirect pathway and GPi-SNr input from direct pathway diminishes. These changes and other variations which are caused by BG destruction are assimilated very well by the model.

Table 1 shows connection weights; each number shows Euclidean norm of relative connection weights. Positive numbers show increasing and negative ones decreasing trend in the amount of neurotransmitters. Higher values demonstrate more neurotransmitters change in connection weights. Vast neuron destruction in indirect pathway makes related values bigger and causes enormous difference between them that clearly represented in striatum to GPe and GPe to STN connections (Table 1). These changes are in accordance with the changes in HD states (Figure 2) and progress with HD severity. We can conclude that alterations caused in the proposed model like neural destruction and neurotransmitters changes in HD state are completely matched with physiological findings.

Fine similarity between the presented model and BG physiological structure with its high ability in simulating HD disorders, introduces this model as a powerful tool to analyze HD behavior. This great ability in simulating could be used to evaluate effects of various drugs on neurotransmitter changes and propose some new medications. Also it could use to evaluate other nonpharmaceutical treatments of HD like deep brain stimulation. This model can rapidly and cheaply assess different hypothesis; so it could be use on clinical research to find novel treatment methods. Moreover by little change in connection weights, the model generates numerous HD behaviors that could be used in quantifying studies, anticipating the BG destruction process, predicting psychiatric symptoms, and finally prescribing the suitable dosage of drugs.

\section{Reference}

Aziz, W., \& Arif, M. (2006). Complexity analysis of stride interval time series by threshold dependent symbolic entropy. European Journal of Applied Physiology, 98(1), 30-40.

Banaie, M., Pooyan, M., \& Mikaeili, M. (2011). Introduction and application of an automatic gait recognition method to diagnose movement disorders that arose of similar causes. Expert Systems with Applications, 38(6), 7359-7363.

Banaie, M., Sarbaz, Y., Gharib-Zadeh, Sh., \& Towhidkhah, F. (2008). Huntington's disease: Modeling the gait disorder and proposing novel treatments. Journal of Theoretical Biology, 254(2), 361-367.

Daliri, M. R. (2012). Automatic diagnosis of neuro-degenerative diseases using gait dynamics. Measurement, 45(7), 1729-1734.

Dutta, S., Chatterjee, A., \& Munshi, S. (2009). An automated hierarchical gait pattern identification tool employing cross-correlationbased feature extraction and recurrent neural network based classification. Expert Systems, 26(2), 202-217.

Fenneya, A., Jogb, M. S., \& Duval, C. (2008). Bradykinesia is not a "systematic" feature of adult-onset Huntington's disease; implications for basal ganglia pathophysiology. Brain Research, 1193, 67-75.

Galvan, A., \& Smith, Y. (2010). Basal Ganglia. In K. Kompoliti \& L. Verhagen (Eds.), Encyclopedia of Movement Disorders (vol. 1, pp. 113-118). San Diego, CA: Academic Press.

Hausdorff, J. M., Cudkowicz, M. E., Firtion, R., Wei, J. Y., \& Goldberger, A. L. (1998). Gait variability and basal ganglia disorders: Stride-to-stride variations of gait cycle timing in Parkinson's disease and Huntington's disease. Movement Disorders, 13(3), 428437

Hausdorff, J. M., Mitchell, S. L., Firtion, R., Peng, C. K., Cudkowicz, M. E., Wei, J. Y., et al. (1997). Altered fractal dynamics of gait: Reduced stride-interval correlations with aging and Huntington's disease. Journal of Applied Physiology, 82(1), 262-269.

Kandel, E. R., Schwartz, J. H., \& Jessell, T. M. (2000). Principles of Neural Science (4 $4^{\text {th }}$ ed.). New York: McGraw-Hill.

Kliem, M. A., \& Wichmann, T. (2009). Basal Ganglia: Functional Models of Normal and Disease States. In P. R Hof \& C. V Mobbs (Eds.), Encyclopedia of Neuroscience (pp. 229-233). Amsterdam, Springer: Academic Press.

Liao, F., Wang, J., \& He, P. (2008). Multi-resolution entropy analysis of gait symmetry in neurological degenerative diseases and amyotrophic lateral sclerosis. Medical Engineering \& Physics, 30(3), 299-310.

Mandic, D., \& Chambers, J. (2001). Recurrent Neural Networks for Prediction: Learning Algorithms, Architectures and Stability. New York: John Wiley \& Sons, Inc. 
Merrikh-Bayat, F. (2011). Time series analysis of Parkinson's disease, Huntington's disease and amyotrophic lateral sclerosis. Procedia Computer Science, 3, 210-215.

Sarbaz, Y., Banaei, M., \& Gharib-Zadeh, S. (2007). A computational model for the Huntington disease. Medical Hypotheses, 68, 11541158 .

Sarbaz, Y., Gharib-Zadeh, S., Towhidkhah, F., Banaie, M., \& Jafari, A. (2011). A gray-box neural network model of Parkinson's disease using gait signal. Basic and Clinical Neurosciences, 2(3), 33-42.

Sarbaz, Y., \& Pourhedayat, A. (2013). Spectral analysis of gait disorders in Huntington's disease: A new horizon to early diagnosis. Journal of Mechanics in Medicine and Biology, 14(1), 1450001.

Sarbaz, Y., Towhidkhah, F., Banaei, M., Pooyan, M., \& Gharib-Zadeh, Sh. (2011). Modelling the gait of normal and Parkinsonian persons for improving the diagnosis. Neuroscience Letters, 509(2), $72-75$.

Sarbaz, Y., Towhidkhah, F., Gharib-Zadeh, S., \& Jafari, A. (2012) Gait spectral analysis: An easy fast quantitative method for diagnosing Parkinson's disease. Journal of Mechanics in Medicine and Biology, 12(3), 1250041

Sarbaz, Y., Towhidkhah, F., Mosavari, V., Janani, A., \& SoltanZadeh, A. (2013). Separating Parkinsonian patients from normal persons using handwriting features. Journal of Mechanics in Medicine and Biology, 13(3), 1350030.

Too, H. K. (1999). Statistical design of experiments using multilayer perceptron neural networks. San Diego: University of California.

Vonsattel, J. G., Keller, C., \& Amaya, M. P. (2008). Neuropathology of Huntington's disease. Handbook of Clinical Neurology, 89, 599618.

Zheng, H., Yang, M., Wang, H., \& McClean, S. (2009). Machine learning and statistical approaches to support the discrimination of neuro-degenerative diseases based on gait analysis. In Intelligent Patient Management (pp. 57-70). Berlin, Springer: Heidelberg. 